\title{
Performance Evaluation of AODV and DSDV Routing Protocols over Zigbee Network for Different Topologies under CBR Traffic Pattern
}

\author{
Smriti Maurya \\ M.E. Scholar, \\ CSE Dept. \\ M.B.M. Engineering College, J.N.V. University,
Jodhpur
}

\author{
N.C. Barwar, PhD \\ Assoc. Professor, \\ CSE Dept. \\ M.B.M. Engineering College, J.N.V. University, \\ Jodhpur
}

\begin{abstract}
Zigbee is an open specification developed by Zigbee Alliance build on the top of IEEE802.15.4 Physical and Media Access Control layer standard, which is one of the global wireless standards of communication protocol for Low-Rate Wireless Personal Area Networks (LR-WPAN). It aims at low power consumption, low data rate, low cost, short range and flexible, reliable, scalable wireless communication. This paper presents overview of IEEE 802.15.4/Zigbee and analyze the performance of AODV and DSDV routing protocols over Zigbee network for different topologies like Random, Star and Peer to Peer (Mesh) under CBR traffic pattern by varying number of nodes, number of sources and range. Performance analysis is carried out using various parameters like Average End-to-End delay, Average Throughput and Packet delivery ratio to determine the Quality of Services (QoS) of the network under different scenarios using NS2 simulator (Version 2.35). Awk scripts are used for analyzing the simulation results and results are shown in graphical forms.
\end{abstract}

\section{General Terms}

IEEE802.15.4/Zigbee Lower rate wireless personal area network

\section{Keywords}

Zigbee, AODV, DSDV, CBR

\section{INTRODUCTION}

With the rapid growth of mobile computing devices the demand for short range wireless standards for portable and flexible connectivity are increasing day by day. Therefore, IEEE802.15 working group was formed in January 1999 to create Wireless Personal Area Network (WPAN) standard, intended to focus on low-cost, low power, small network and short range. The idea was to create standards that allow devices to communicate and collaborate with one another and to accommodate wider adoption and applicability.

Zigbee is an open specification for Lower rate wireless personal area network introduced by Zigbee Alliance in 2003. IEEE and ZigBee alliance have joined hands to develop a complete specification of protocol stack for IEEE802.15.4. Zigbee is built on the top of IEEE802.15.4 standard where IEEE802.15.4 standard focuses on the specification of the lower two layers (physical and data link layer) that supports for low data rate, low power consumption and low cost and ZigBee works on upper layers of the protocol stack (network, and application layer) that enables interoperable data networking and security services. Normally, ZigBee works in the license-free $2.4 \mathrm{GHz}$ ISM band with a data rate of $250 \mathrm{Kbps}$ and the transmission distance ranges from 10 to 75 meters, which makes this technology easily applicable and worldwide available. In LR-WPAN, a device can be assigned either a 16 bit short or a 64 bit extended address during association. Hence, a single network could potentially accommodate $65,536\left(2^{16}\right)$ devices. It is anticipated to enable various short range applications in the fields of home networking, home/office automation, automotive networks, industrial networks, hotels, hospitals, interactive toys and remote metering and many more [6]. Before the implementation of real time applications, extensive performance evaluation of the standard is necessary especially when critical issue like QoS is of concern. The knowledge of traffic patterns is also essential for optimizing the performance because the uneven distribution make traffic heterogeneous in nature and it will affects the QoS. So it's a challenge to choose the optimal routing protocol that can be used with most usable data traffic pattern to enhance the QoS of network. In this paper an attempt is made to analyze the performance of AODV and DSDV routing protocols over IEEE802.15.4/Zigbee network for different topologies under CBR Traffic pattern with respect to the related work done by various researchers.

The rest of the paper is organized as follows section II covers related work in this area. Section III provides a brief overview of the IEEE802.15.4/ Zigbee standard. Section IV describes various routing protocols and traffic patterns, in section $\mathrm{V}$ simulation environment is discussed, simulation results are analyzed in section VI and finally section VII gives conclusion of the paper.

\section{RELATED WORK}

Several efforts on performance evaluations were conducted since the inception of IEEE802.15.4. The literature comprehensively defines the IEEE802.15.4 protocol as well as simulations on different traffic patterns and different topologies using AODV and DSDV routing protocols. Some of them are discussed below.

J.Zheng and M.J.Lee [1] developed the IEEE 802.15.4 standard on the ns 2 simulator and carry out several sets of experiments to study its various features.

Prathamesh Ajgaonkar et al. [2] conduct AODV simulation with different traffic scenarios like FTP, CBR and Poisson, having different network topologies (Star and Peer to Peer on 
various types of queue such as Drop Tail, Stochastic Fair Queue (SFQ), and Random Early Detection (RED) is carried out to analyze QoS parameters like end-to-end packet delay and jitter.

Elmustafa Sayed Ali Ahmed et al. [3] identify the performance of data traffic patterns (CBR, FTP and POISSON) in Zigbee personal area networks (PAN) using MANET routing protocols (AODV, DSR and INTANTSENSE).Simulation and computations of throughput ,data loss, PDR, overhead and delay done using NS2 simulator (Version 2.34) with parameter of quality of data and pause time.

Arpitha et al. [4] analyze the performance of IEEE 802.15.4 topologies such as star, mesh and cluster tree of WPAN using different performance metrics like goodput, throughput, endto-end delay with respect to routing protocol AODV and DSDV using NS2.

Deepali Ramesh et al. [5] provides a brief description of Zigbee standards focusing on developing different topology models using AODV protocol and using different traffics to analyze using various metrics like packet delivery ratio, jitter, end to end delay and load factor using NS2.

Chavan et al. [6] evaluate ZigBee based WPAN by considering PDR, Throughput, Jitter and Delay, for Star and mesh topologies and generate various scenarios by varying number of node on mobility, range, routing and simulation time.

Veerendra et al. [7] perform simulation for three network topology (scatternet, piconet and peer to peer) with varying network density using both beacon and non-beacon enabled mode on different traffic type.

Gowrishankar.S et al. [8] evaluate IEEE 802.15.4 over AODV with sink mobility through variations in traffic load, number of source nodes and packet size. Various metrics like PDR, Network Throughput, Average Network Delay and Normalized Routing Load is considered for evaluation.

Sangeetha et al. [9] analyze IEEE 802.15.4/ZigBee network for AODV and DSDV protocols by using throughput and energy remaining per node as the performance metrics using NS2 simulator.

Haithem et al. [10] analyze AODV and DSR performance for IEEE 802.15.4/ZigBee using network simulator Ns2 based on packet loss, network throughput packet delivery ratio, energy consumption and average delay. Various simulation scenarios by varying network and traffic densities are investigated.

\section{OVERVIEW OF IEEE802.15.4/ ZIGBEE}

A brief overview of IEEE802.15.4/Zigbee standard, focusing on the details relevant to the performance study is given in this section. The IEEE802.15.4/Zigbee protocol Stack is shown in Fig $1[1,18,19]$. The IEEE802.15.4 forms the basis for the ZigBee specification. IEEE focuses on the lower two layers of the protocol (physical and Mac layer) whereas ZigBee works on upper layers of the protocol stack (network, and application layer).

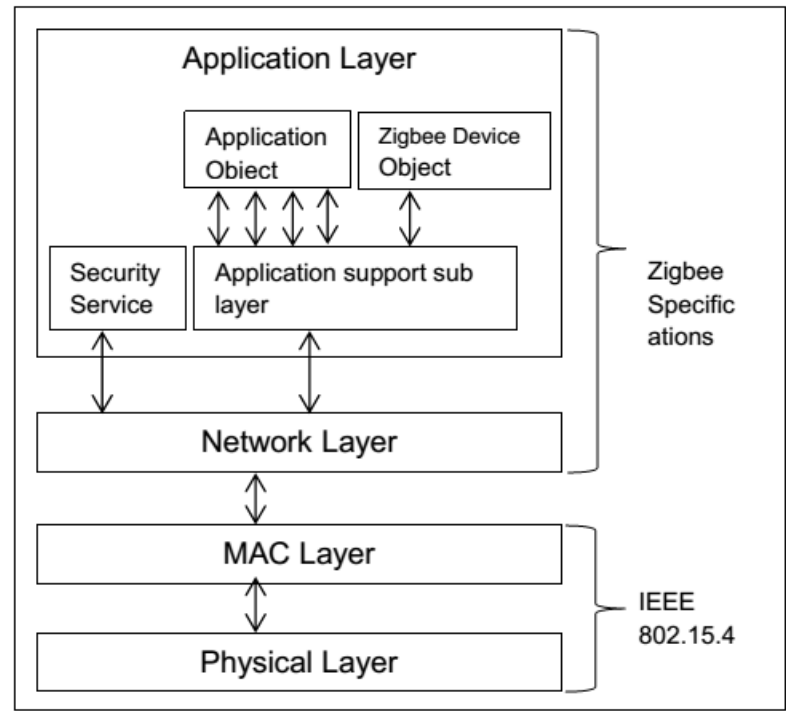

Fig 1: Layered Protocol Stack of IEEE 802.15.4

The Physical layer defined by IEEE802.15.4 is responsible for activation and deactivation of the radio transceiver, energy detection (ED), link quality indication (LQI), channel selection, clear channel assessment (CCA) and transmitting as well as receiving packets across the physical medium. At the physical layer, Zigbee operates on three different frequency bands $2.4 \mathrm{GHz}, 915 \mathrm{MHz}$ and $868 \mathrm{MHz}$ 's [15]. The supported data rate is $250 \mathrm{kbps}$ at $2.4 \mathrm{GHz}$ with offset quadrature phase shift keying (OQPSK) modulation, $40 \mathrm{kbps}$ at $915 \mathrm{MHz}$ and $20 \mathrm{kbps}$ at $868 \mathrm{MHz}$ with binary phase shift keying (BPSK) modulation. Total of 27 channels is utilized which includes 1 channel between 868 and $868.6 \mathrm{MHz}, 10$ channels between 902.0 and $928.04 \mathrm{MHz}$ and 16 channels between 2.4 and $2.4835 \mathrm{GHz}$. Several channels in different frequency bands make it possible to relocate within the available spectrum.

The MAC layer defined by IEEE 802.15 .4 controls the access to the communication channel and provides flow control through acknowledgements and retransmissions. It also controls frame validation, beacon management, device synchronization, guarantees time slot management and handles nodes association and disassociation.

The MAC layer defines two types of devices a full-function device (FFD) and a reduced-function device (RFD). The FFD can operate as a PAN coordinator, a coordinator or a device. An FFD can communicate with RFDs or other FFDs, while an RFD can only communicate with the FFD. RFD is intended for very simple applications that do not require the transfer of large amounts of data and need minimal resources.

The MAC layer supports two operational modes beaconenabled mode and non-beacon-enabled mode. Beacon enabled mode is used when the coordinator runs on batteries and thus offers maximum power savings, whereas the non-beacon mode is used when the coordinator is mains-powered.

In beacon enabled mode, PAN coordinator broadcasts beacons periodically to synchronize the attached devices for effective communication. In this mode superframe structure $[16,19]$ is used for communication over network as shown in Fig 2 which has a beacon on either side of the structure. Each superframe structure consists of active and inactive period. In active period communication takes place and nodes send 
packets using slotted CSMA-CA mechanism. Superframe consists of a beacon, a contention access period (CAP) and a contention free period (CFP). In CAP, the device must compete with other devices. In the CFP, the PAN coordinator assigns guaranteed time slots (GTS) to a single device, which together forms the CFP. In an inactive period node is tuned off to save the battery power. The structure of superframe is characterized by two parameters Superframe Order (SO) and Beacon Order (BO). SO is a variable which is used to determine the length of the superframe duration and $\mathrm{BO}$ is a variable which is used to establish the Beacon Interval (BI) which defines the length of superframe. The BI and SD can be defined as follows:

$\mathrm{BI}=\mathrm{B} \times 2 \mathrm{BO}$

$\mathrm{SD}=\mathrm{B} \times 2 \mathrm{SO}$

Constant B can be defined as a base superframe duration which is a minimum duration of superframe when the value of $\mathrm{BO}$ is equal to 0 . The relation between $\mathrm{SO}$ and $\mathrm{BO}$ that must be satisfied is given as:

$0 \leq \mathrm{SO} \leq 14$

$0 \leq \mathrm{BO} \leq 14$

When $\mathrm{BO}=14$ then there are no beacon transmissions. An inactive portion is denoted, when the beacon interval is same as that of the superframe duration $(\mathrm{SO}=\mathrm{BO})$ and when beacon order is greater than superframe order.

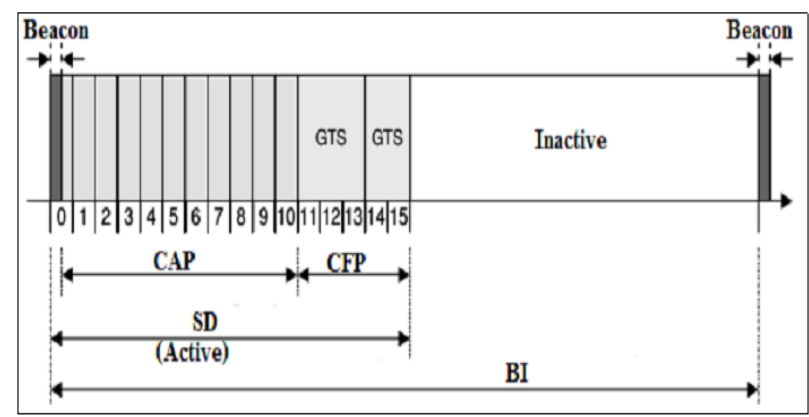

Fig 2: Superframe Structure of IEEE 802.15.4 in an Beacon Enabled mode

In nonbeacon-enabled mode, PAN coordinator does not broadcast beacons periodically and communication is carried out using unslotted CSMA. It is useful whenever there is light traffic in between nodes. The power consumption is more in this mode as compared to beacon enabled mode as the node keeps on listening channel until it is found idle and transmission starts when channel is idle so various nodes compete to access the channel.

Different topologies supported by IEEE802.15.4 are star, peer to peer (Mesh) and cluster tree topologies $[18,19]$ as is shown in Fig 3.

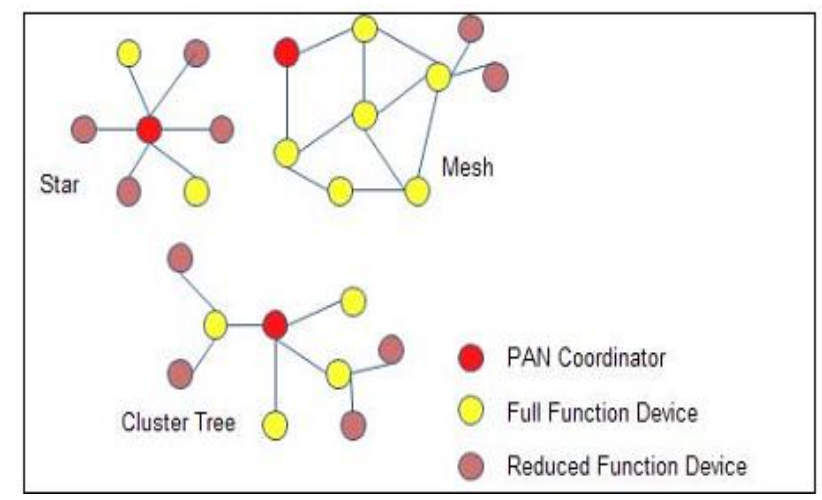

Fig 3: Zigbee Topology Models

Peer-to-peer (Mesh) networks capable of performing selfmanagement and organization form arbitrary patterns of connections. In a star network all the end devices, directly communicate with the coordinator. Cluster-tree network is a special case of a peer-to-peer network in which most devices are FFDs and an RFD. It consists of a number of clusters connected whose central nodes are also in direct communications with the single PAN Coordinator.

Network layer is the lowest layer of ZigBee and acts as an interface between application layer and MAC Layer. It is responsible for starting a network, joining and leaving a network, configuring a new device, addressing, topology specific routing, neighbor's discovery and routing discovery.

Application Layer is the highest layer of the ZigBee stack. ZigBee specification divides the layer into three different sublayers: Application Support Sub layer, ZigBee Device Objects and manufacturer defined Application Objects. It is responsible for grouping address definitions, fragmentation and reassembly of packets, reliable data transports, establishing and managing the cryptographic keys for security, maintaining tables for binding and forwarding messages between devices.

\section{OVERVIEW OF ROUTING PROTOCOLS AND TRAFFIC PATTERNS}

\subsection{Routing Protocols}

There are two main approaches for routing process in ad-hoc networks. The first approach is a proactive or Table-driven approach which determines the layout of the network and maintain consistent and up-to date routing information by periodically distributing routing information among each other. Proactive protocols present low latency, but high routing overhead, as the nodes periodically exchange control messages and routing-table information in order to keep up todate route to any active node in the network. The second approach is Reactive, source-initiated or on-demand approach. Reactive protocols create routes as and when required by using route discovery mechanism to reduce traffic overhead. Reactive protocols do not maintain up-to-date routes to any destination in the network and do not generally exchange any periodic control messages. Thus, they present low routing overhead, but high latency as compared to proactive protocols. The DSDV is a proactive protocol and AODV, DSR, and TORA are reactive protocols. The ad-hoc routing protocols considered in this study are described below [23]. 


\subsubsection{Destination Sequenced Distance Vector (DSDV)}

Destination Sequenced Distance Vector (DSDV) is a loop free routing protocol in which the shortest path is calculated based on the Bellman-Ford algorithm. Data packets are transmitted between the nodes using routing tables stored at each node. Each routing table contains all the possible destinations from a node to any other node in the network and also the number of hops to each destination. The protocol has three main attributes: to avoid loops, to resolve the "count to infinity" problem and to reduce high routing overhead. Each node issues a sequence number that is attached to every new routing-table update message and uses two different types of routing-table updates, named "full" and "incremental dumps", respectively, to minimize the number of control messages disseminated in the network. Each node keeps statistical data concerning the average setting time of a message that the node receives from any neighboring node. The data is used to reduce the number of rebroadcasts of possible routing entries that may arrive at a node from different paths but with the same sequence number. DSDV takes into account only bidirectional links between nodes [14].

\subsubsection{Adhoc On Demand Distance Vector Routing Algorithm (AODV)}

Adhoc On Demand Distance Vector Routing Algorithm (AODV) uses routing tables which stores, one entry per destination. It is capable of both unicast and multicast routing. To find a route to the destination, the source node floods the network with RouteRequest packets (RREQ), and the packets carry the destination address and sequence number. The RouteRequest packets create temporary route entries for the reverse path through every node it passes in the network. When it reaches the destination a RouteReply (RREP) is sent back through the same path the RouteRequest was transmitted. Every node maintains a route table entry which updates the route expiry time. A route is valid for the given expiry time, after which the route entry is deleted from the routing table. Whenever a route is used to forward the data packet the route expiry time is updated to the current time plus the Active Route Timeout. An active neighbor node list is used by AODV at each node as a route entry to keep track of the neighboring nodes that are using the entry to route data packets. These nodes are notified with RouteError packets when the link to the next hop node is broken. Each such neighbor node, in turn, forwards the RouteError to its own list of active neighbors, thus invalidating all the routes using the broken link. AODV is designed to support communication between mobile nodes with lowest possible routing path.

\subsection{Traffic Patterns}

Data source of any network can be modeled using various traffic patterns. Traffic models are mainly used for prediction of performance of network and congestion of network. Traffic model should be such that it resembles real world network traffic. It should satisfy specific applications of a network and enhance the capacity of a network. The knowledge of communication pattern and traffic characteristics is essential for designing and optimizing its performance. The development of universal traffic model is not possible because it may appropriate for one application but not suitable for another application.
There are three data traffic patterns CBR, FTP and Poisson which are mostly used in the networks $[3,13]$.

\subsubsection{Constant Bit Rate (CBR)}

The Constant Bit Rate (CBR) Traffic is a real time traffic that consume and constant the sending rate, where the traffic sending rate is specified at the Peak Cell Rate (PCR) parameter. CBR traffic model provides the best guarantee of delivery of traffic. It uses UDP as its transport agent.

\subsubsection{File Transfer Protocol (FTP)}

File Transfer Protocol (FTP) is a standard mechanism provided by the Internet for transferring files from one host to another. File transfer protocol is the application protocol based on client server model and is used for transfer of web pages, to download programs and other files.

\subsubsection{Poisson}

Poisson generates traffic when bit rate is variable and this traffic model is suitable when data traffic is not bursty. It generates traffic according to Poisson distribution. Packets are sent at very high rate during on period and no packets are sent during off period. Packets are of constant size.

\section{SIMULATION ENVIRONMENT}

Simulation is carried out using network simulator tool in which Zigbee uses operating frequency of $2.4 \mathrm{GHz}$ with maximum data rate of $250 \mathrm{kbps}$. Network Simulator (Version 2 ), widely known as NS2 is an open source event driven simulation tool designed specifically for research in communication networks that simulates the behavior of network without an actual network is being present. It is an object oriented simulator written in $\mathrm{OTcl}$ and $\mathrm{C}++$ languages. $\mathrm{C}++$ is for backend used to run simulation and OTcl is for frontend used to create and configure the network. After simulation, NS2 outputs either text based or animation-based simulation results. To interpret these results graphically and interactively, tools such as NAM (Network AniMator) and XGraph are used.

\subsection{Performance metrics}

Performance of Zigbee protocol is evaluated using the following metrics:

\subsubsection{Packet Delivery Ratio:}

It is the ratio of data packets successfully delivered to the destination to the data packets generated by the source. A high value of Packet Delivery Fraction indicates that most of the packets are being delivered to the higher layers and is a good indicator of the protocol performance

\subsubsection{Average End-to-End Delay:}

This is the average time delay for data packets from the source node to the destination node. Ideally the value of end to-end delay should be as low as possible.

\subsubsection{Average Throughput:}

The successful transmission of data packets in a unit time is known as throughput. It is usually measured in kbps. It should be high. 


\subsection{Experimental Setup}

The various simulation parameters used is shown in Table 1 .

Table 1. Simulation Parameters

\begin{tabular}{|c|c|}
\hline Parameter & Value \\
\hline Channel Type & Wireless Channel \\
\hline MAC Protocol & IEEE 802.15 .4 \\
\hline Routing Protocol & AODV, DSDV \\
\hline Network Topology & $\begin{array}{c}\text { Random, Star, Peer to Peer } \\
\text { (Mesh) }\end{array}$ \\
\hline Terrain Size & 80 X80 m ${ }^{2}$ \\
\hline Traffic Type & CBR \\
\hline Simulation Time(s) & 100 \\
\hline Number of Nodes & $10,20,30,40,50$ \\
\hline Number of sources & $1,3,5,7$ \\
\hline Range(m) & $10,15,20,25$ \\
\hline Queue Type & Drop Tail \\
\hline Radio propagation models & Two ray ground \\
\hline Antenna Model & Omni-Directional Antenna \\
\hline $\begin{array}{c}\text { Beacon order, Super frame } \\
\text { order }\end{array}$ & 3 \\
\hline
\end{tabular}

Three set of simulation is carried out using CBR Traffic pattern and snapshot of NAM window for simulated ZigBee network with 10 nodes for Random, Star and Peer to Peer (Mesh) topology is shown in Fig 4, Fig 5 and Fig 6 respectively. In the First set a ZigBee network with Random topology, in the second set a Zigbee network with Star topology and in third set a Zigbee network with Peer to Peer (Mesh) topology has been set up and analyzed by varying Number of nodes, Number of sources and Range between nodes. When Number of nodes is varied Number of sources 3 and Range $10 \mathrm{~m}$ is kept constant, when Number of sources is varied Number of nodes 20 and Range $20 \mathrm{~m}$ is kept constant and when Range is varied Number of nodes 20 and Number of sources 3 is kept constant. The network is simulated using both AODV and DSDV protocols. The combinations of the nodes are chosen randomly and user can make the selection of any source and destination pair.

\subsubsection{AODV / DSDV simulation for Random Topology}
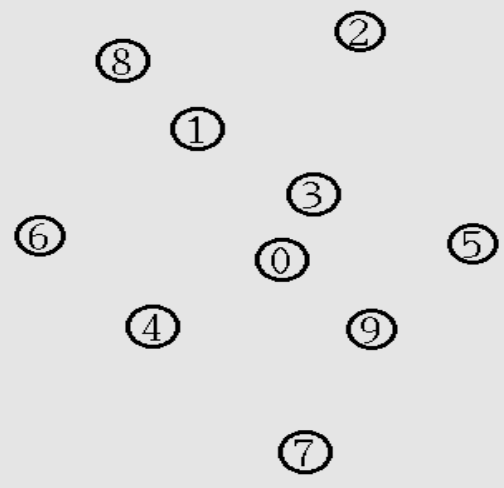

Fig 4: Snapshot of Random Topology

\subsubsection{AODV/DSDV simulation for Star Topology}

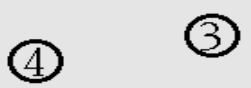

(2)

(5)

(Q)

(1)

(6)

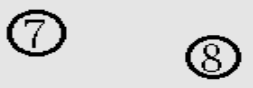

(9)

Fig 5: Snapshot of Star Topology

\subsubsection{AODV / DSDV simulation for Peer to Peer (Mesh) Topology}

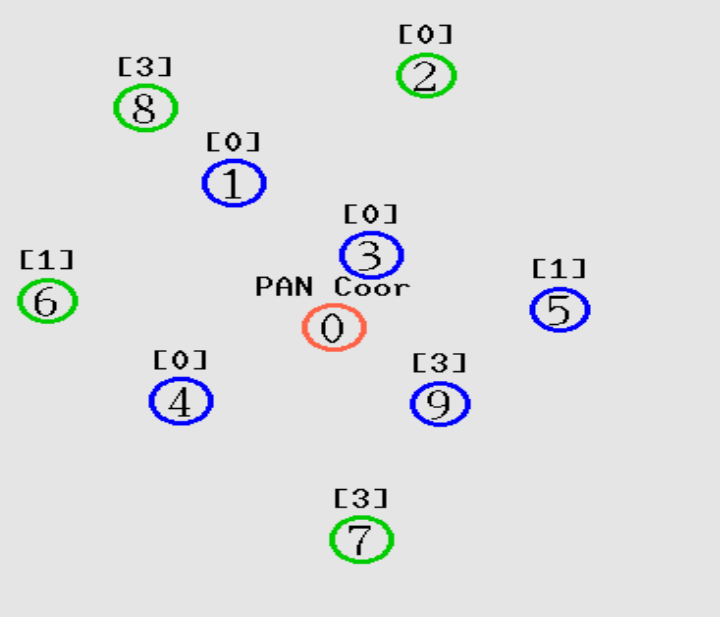

Fig 6: Snapshot of Peer to Peer (Mesh) Topology

The trace file generated after simulation is shown in Fig 7 for CBR traffic Pattern.

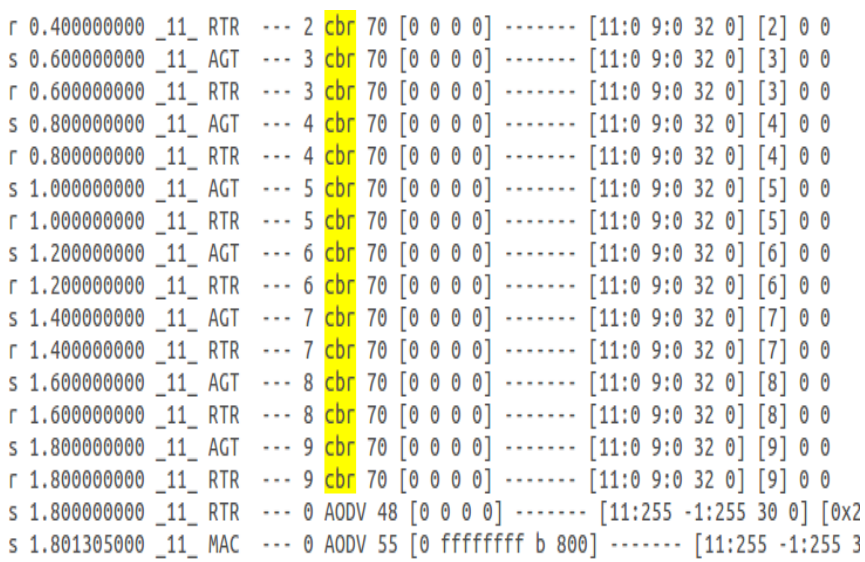

Fig 7: Snapshot of Trace file after Simulation for CBR traffic pattern 


\section{SIMULATION RESULTS ANALYSIS}

In this section the performance of IEEE 802.15.4/Zigbee for different topologies using AODV and DSDV routing protocols over CBR Traffic Pattern by varying Number of nodes, Number of sources and Range has been studied through extensive simulation.

\subsection{Packet Delivery Ratio}

Fig 8, Fig 9 and Fig 10 shows the performance of Packet Delivery Ratio for Random, Star and Peer to Peer (Mesh) topology over AODV and DSDV Protocols by varying Number of nodes, Number of sources and Range respectively.

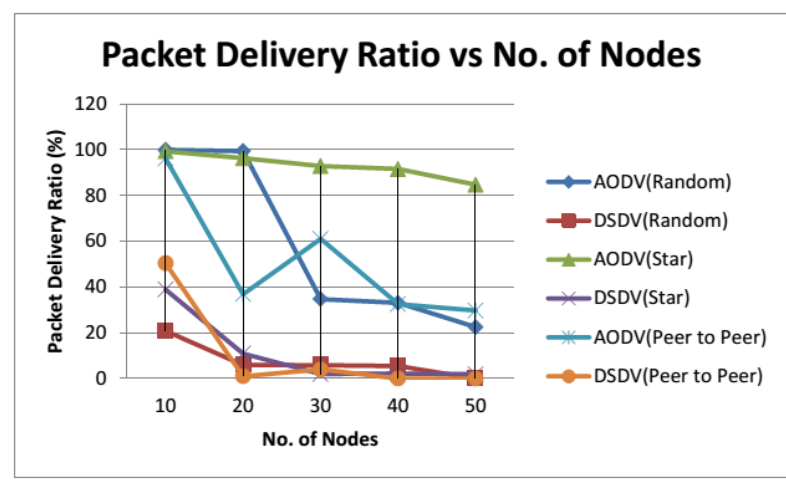

Fig 8: Packet Delivery Ratio vs No. of Nodes

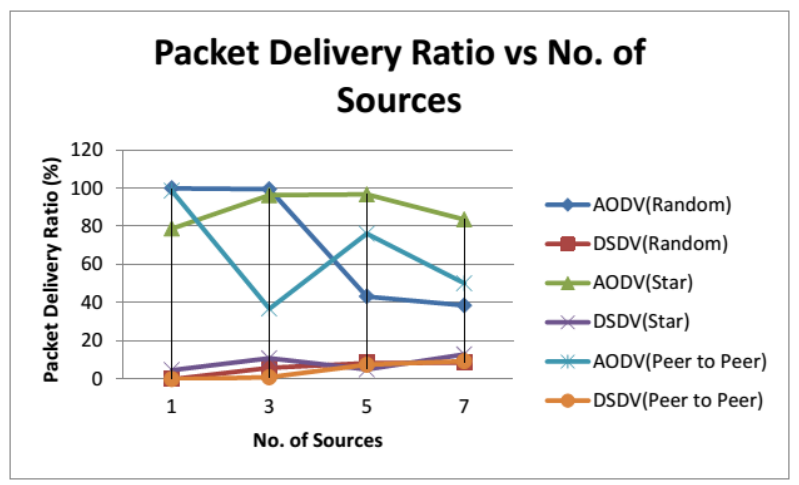

Fig 9: Packet Delivery Ratio vs No. of Sources

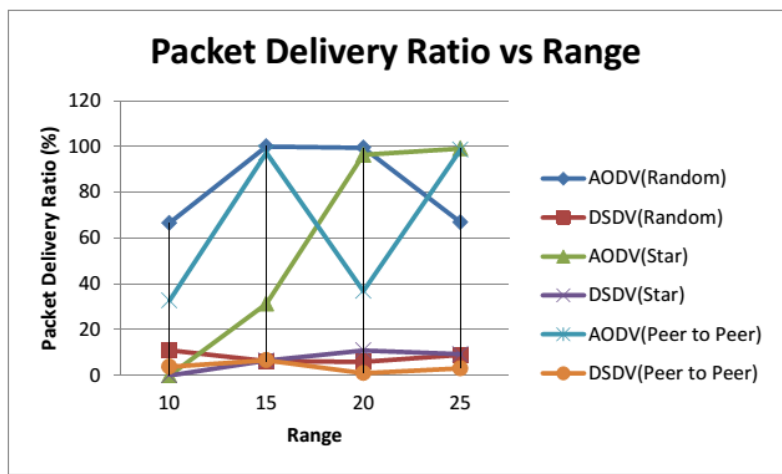

Fig 10: Packet Delivery Ratio vs Range

It is shown in Fig 8, Fig 9 and Fig 10 that Packet delivery ratio of AODV is better than DSDV for all scenarios. The reason for DSDV's low packet delivery ratio is that it is a table-driven protocol and updates its table periodically which leads to an increase in the routing load in the network and less packet delivery ratio. On the other hand, AODV is an on demand routing protocol and adapts faster than DSDV due to the frequent change of position of nodes. For that AODV can find an alternate route if the current link has broken whereas DSDV fails at that point.

As the Number of nodes increases in the network PDR decreases for both protocols, as the Number of sources increases PDR decreases for AODV and increases for DSDV and as the Range increases, both protocols shows a considerable performance.

\subsection{Average End to End Delay}

Fig 11, Fig 12 and Fig 13 shows the performance of Average End to End Delay for Random, Star and Peer to Peer (Mesh) topology over AODV and DSDV Protocols by varying Number of nodes, Number of sources and Range respectively.

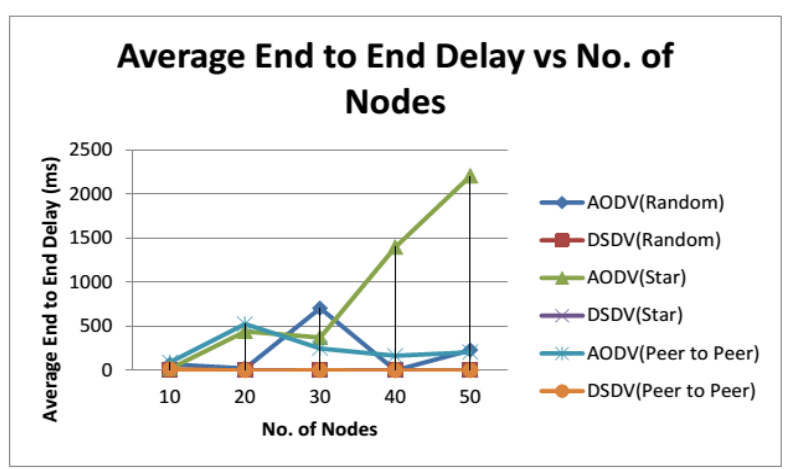

Fig 11: Average End to End Delay vs No. of Nodes

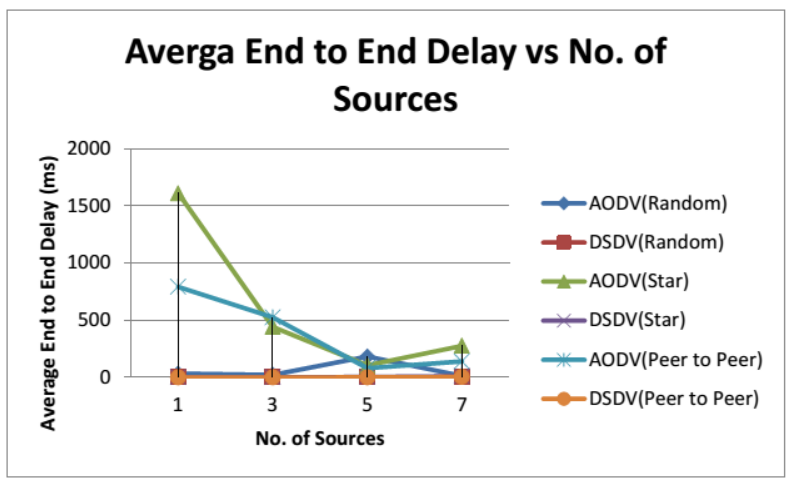

Fig 12: Average End to End Delay vs No. of Sources

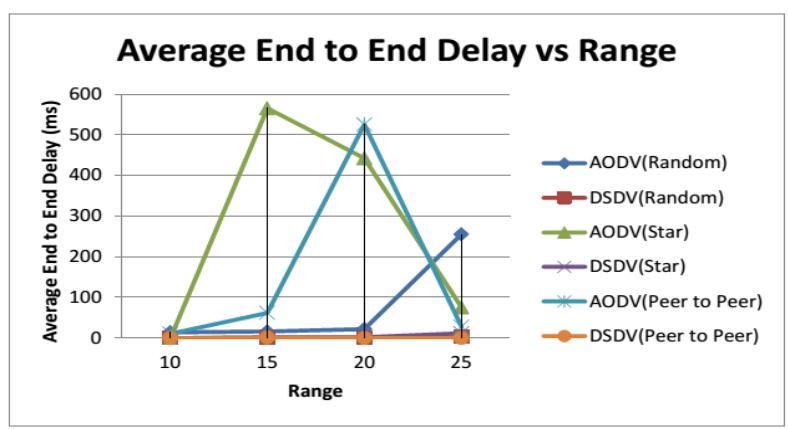

Fig 13: Average End to End Delay vs Range 
It is shown in Fig 11, Fig 12 and Fig 13 that Average End to End delay of AODV is more than DSDV for all scenarios. So DSDV performs much better because of its proactive nature in which the path to a destination is immediately available and no delay is caused by routing discovery. End-to-end delay includes the delay in the send buffer, the delay in the interface queue, the bandwidth contention delay at the MAC, and the propagation delay. Furthermore, DSDV routing protocol drop the packets, if it is not possible to deliver them which means less delay. On the other hand, AODV packets stay in the send buffer till the route is discovered in order to be sent to the destination on that route. The presence of more number of nodes between source and destination affects the increase in hop count thus resulting in increased average end-to-end delay. If any link breaks in the current topology, AODV would try to find an alternate path from among the backup routes between the source and the destination node pairs resulting in additional delay to the packet delivery time. In comparison, if any link break occurs in DSDV, the packet would not reach the destination due to unavailability of another path from source to destination.

As the Number of nodes, Number of sources and Range increases both protocols show considerable performance.

\subsection{Average Throughput}

Fig 14, Fig 15 and Fig 16 shows the performance of Average Throughput for Random, Star and Peer to Peer (Mesh) topology over AODV and DSDV Protocols by varying Number of nodes, Number of sources and Range respectively.

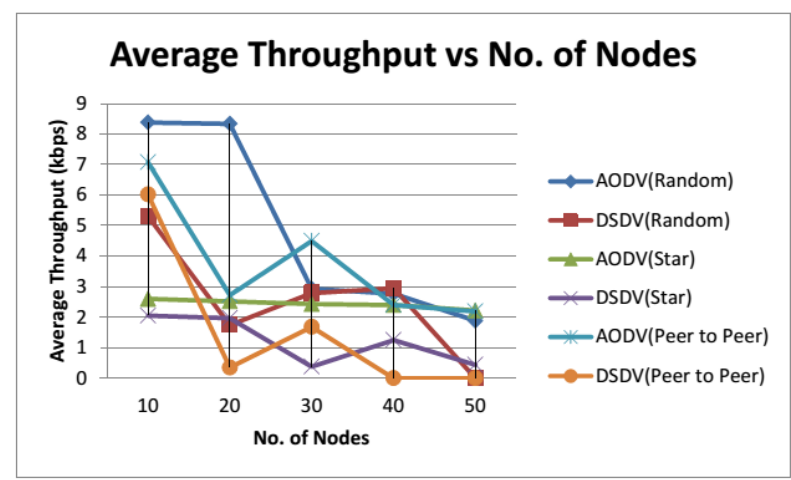

Fig 14: Average Throughput vs No. of Nodes

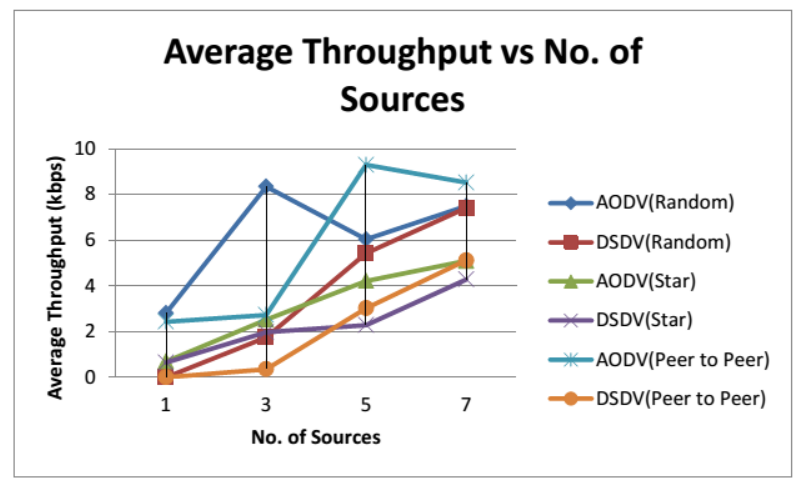

Fig 15: Average Throughput vs No. of Sources

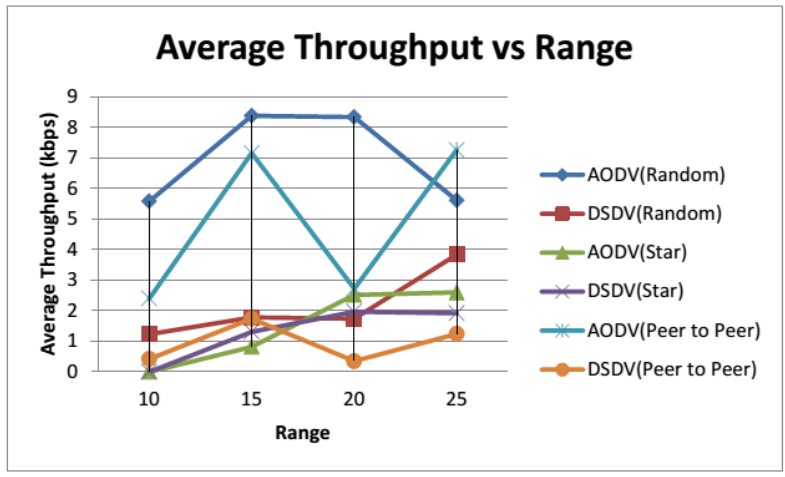

Fig 16: Average Throughput vs Range

It is shown in Fig 14, Fig 15 and Fig 16 that Throughput of AODV is better than DSDV for all scenarios. The reason for AODV's better performance is its reactive nature in which maximum amount of TCP packets are sent and receive from source to destination. It drops a considerable number of packets during the route discovery phase and is more reliable. DSDV routing table update mechanism is not fast enough to update the routing tables when topology changes occur and network congestion occurs due to high traffic in the network because of increase in overhead and control messages for routing updations.

As the Number of nodes increases in the network throughput decreases, as the Number of sources increases throughput increases and as the Range increases both protocols shows considerable performance.

\section{CONCLUSION}

This work is carried out with different parameters using NS2 simulator to study and analyze the performance of AODV and DSDV protocols for different Topologies of Zigbee Network under CBR Traffic patterns considering variation in Number of nodes, Number of sources and Range. Performance is analyzed in terms of packet delivery ratio, average end-to-end delay and average throughput.

Based on the results obtained from the experiments, it is observed that Packet delivery ratio and Average throughput of AODV protocol is better than DSDV but Average End to End delay of DSDV protocol is better. As the Nodes, Sources and Range increases, PDR decreases rapidly for Zigbee network and end to end delay increases this in turn affects throughput. AODV for small network and less congested network might not perform well; in that case DSDV performs better. If the topology is considered Peer to Peer (Mesh) topology gives overall better performance as the Number of Nodes, Number of Sources and Range increases. This is due to the multihop transmissions and routing options availability. So, AODV is a best protocol for Zigbee with Mesh Topology for real time implementation according to this study and the simulation results obtained are almost nearer to the theoretical analysis.

Day-to-day new challenges and requirements are coming. So in future, this study can be explored for other routing protocol like DSR and TORA, for other topologies like tree and cluster tree and for other traffic patterns like FTP and POISSON using more advance network simulators. Other performance parameters like Jitter and Routing Overhead can also be considered. Analysis can be done by varying Pause time, Speed, Range and Deployment Area. 


\section{REFERENCES}

[1] Jianliang Zheng and Myung J. Lee "A Comprehensive Performance Study of IEEE 802.15.4" IEEE Press Book, 2004

[2] Prathamesh Ajgaonkar, Lingfeng Wang, and Mansoor Alam, "Simulation Studies on ZigBee Communications for Home Automation and Networking", IEEE 2010.

[3] Elmustafa Sayed Ali Ahmed, Ibrahim Khider Altahir, A. Alrhman Mohammed and Amani Dawod Salih" Performance Analysis of Traffic Patterns over MANET Routing Protocols in Zigbee Personal Area Network" International Journal of Computer Science and Telecommunications Volume 6, Issue 1, January 2015.

[4] Arpitha E M, Ramesh T M "Performance Analysis of Low Rate WPAN Topologies"International Journal of Innovative Research in Computer and Communication Engineering, 2014.

[5] Deepali Ramesh Borade, Shaikh.Mohd. Laeeq, "Performance and Evaluation of IEEE 802.15.4 under Different Topologies with AODV Protocol", Students' Conference on Electrical, Electronics and Computer Science IEEE 2012.

[6] Chavan S.G., Shirsat S.A., "Performance Comparison of Network Topologies in ZigBee Based WPAN", International Journal of Scientific \& Engineering Research, Volume 4, Issue 10, October-2013 ISSN 2229-5518

[7] G R Veerendra, Nandini Prasad K S, Babu N V and Puttamadappa C, "Topology Based Performance Analysis of IEEE 802.15.4 for Wireless Sensor Networks", IJCSNS International Journal of Computer Science and Network Security, VOL.10 No.8, August 2010

[8] Gowrishankar.S, T.G.Basavaraju, Subir Kumar Sarkar, "Simulation Based Analysis of Mobile Sink Speed in Wireless Sensor Networks", Proceedings of the World Congress on Engineering and Computer Science 2010 Vol I WCECS 2010, October 20-22, 2010, San Francisco, USA

[9] Sangeetha C P, Dr. C.D. Suriyakala, "Performance Analysis of IEEE802.15.4/ZigBee Wireless Sensor Networks" International Journal of Engineering Sciences \& Research Technology, April -2014

[10] Haithem Ben Chikha, Amira Makhlouf and Wiem Ghazel, "Performance Analysis of AODV and DSR Routing Protocols for IEEE 802.15.4/ZigBee"
[11] Rajesh kumar dubey and Anamika pathak,"Performance of Routing Protocols for ZigBee Wireless Sensor Network",Journal of Comp. and I.T. Vol. 3,4(3\&1), 99103 (2013).

[12] Ms. Swati V. Birje, Mr. Mahesh Kumbhar, Mr. Raviraj S. Patkar, "Performance Analysis of IEEE802.15.4" International Journal of Advanced Research in Computer Science and Software Engineering, 2013

[13] Pooja Sahu, Mr. Anshul Shrotriya" Performance Evaluation of IEEE 802.15.4 under Different Traffic Conditions" International Journal of Scientific \& Engineering Research, Volume 4, Issue 12, December20131824 ISSN 2229-5518

[14] Patil v.p." Impact of mobility and network load on the performance of reactive and proactive routing protocol in Manet" International Journal of Computer Engineering \& Science, Sept 2012.

[15] M. M. Chandane, S. G. Bhirud, S.V. Bonde," Performance Analysis of IEEE 802.15.4", International Journal of Computer Applications (0975 - 8887) Volume 40- No.5, February 2012

[16] Vaddina Prakash Rao," The simulative Investigation of Zigbee/IEEE802.15.4", [Online] Available: http://www.vaddina.com/pages/01_01_00_00_Zigbee/0 0 _content/zigbee.pdf.

[17] Harleen Kaur Sahota, Sandeep Singh Kang "ZigBee : A Promising Wireless Technology" International Journal of Computer Science and Network (IJCSN) Volume 1, Issue 6, December 2012

[18] Dusan Stevanovic,"Zigbee / IEEE 802.15.4 Standard talk" June 20, 2007

[19] Sinem Coleri Ergen,"ZigBee/IEEE 802.15.4 Summary” September 10, 2004

[20] Information Sciences Institute, "The Network Simulator Ns-2", [Online] Available: http://www.isi.edu/nanam/ns/.

[21] Zigbee Simulations, [Online] Available: "http://www.ifn.et.tudresden.de/ marandin/ZigBee/

[22] ZigBee Alliance, “ZigBee Specification”, Janvier 2008

[23] Baldev Ram Mali, N.C. Barwar"Performance of MANET Routing Protocols considering Impact of Node Density under Different Traffic Patterns" Special Issue of International Journal of Computer Applications (0975 - 8887) on Wireless Communication and Mobile Networks, No.6. Jan.2012, ww.ijcaonline.org 